\title{
Correlation of meta 1 expression with culture stage, cell morphology and infectivity in Leishmania (Leishmania) amazonensis promastigotes
}

\author{
Marcos Gonzaga dos Santos ${ }^{1 /+}$, Maria Fernanda Laranjeira da Silva ${ }^{1}$, \\ Ricardo Andrade Zampieri', Rafaella Marino Lafraia ${ }^{2}$, Lucile Maria Floeter-Winter ${ }^{1}$ \\ ${ }^{1}$ Departamento de Fisiologia, Instituto de Biociências, Universidade de São Paulo, São Paulo, SP, Brasil \\ ${ }^{2}$ Laboratório de Imunoquímica, Instituto Butantan, São Paulo, SP, Brasil
}

The parasitic protozoan Leishmania (Leishmania) amazonensis alternates between mammalian and insect hosts. In the insect host, the parasites proliferate as procyclic promastigotes and then differentiate into metacyclic infective forms. The meta 1 gene is preferentially expressed during metacyclogenesis. Meta 1 expression profile determination along parasite growth curves revealed that the meta $1 \mathrm{mRNA}$ level peaked at the early stationary phase then decreased to an intermediate level. No correlation was observed between meta 1 expression and infectivity. Conversely, infectivity correlated with the increase of apoptotic cells in the late stationary phase.

Key words: meta 1 expression - metacyclic morphology - phosphatidylserine exposure

Leishmania is the causative agent of a wide spectrum of diseases commonly known as leishmaniasis. The parasitic protozoa Leishmania (Leishmania) amazonensis is the causative agent of cutaneous leishmaniasis in South America. The parasites alternate between mammalian and Phlebotominae insect hosts. In the mammalian host, they live as obligate intracellular amastigotes, characterized by round cells with no evident flagellum, in macrophages, where they proliferate in phagolysosomes. The parasites are transmitted between mammalian hosts via the bite of female phlebotomine sand flies. In the gut lumen of the insect host, the proliferative form is the procyclic promastigote, an oblong cell with a flagellum arising from the flagellar pocket at the anterior end of the organism. These cells differentiate into the metacyclic promastigote, the infective stage, which is a small and slender promastigote with a longer flagellum (Bates $\&$ Tetley 1993). They are delivered to a new mammalian host through the next blood feeding of the insect (Descoteaux \& Turco 1999, Cunningham 2002). The mechanisms involved in the differentiation signalling from procyclic to metacyclic promastigotes in an insect host are poorly understood. However, metacyclogenesis occurs in axenic cultures in vitro and can be induced by cultivating promastigotes in an acidic $\mathrm{pH}$ (Sacks \& Perkins 1984, Bates \& Tetley 1993). Differentiation into the metacyclic form induces changes in the cell surface, rendering the cells resistant to complement-mediated lysis and also more infective (Sacks \& Perkins 1984, Franke et al. 1985, Sacks et al. 1985). Some differentiated meta-

Financial support: FAPESP, CNPq

MGS, MFLS and RML received fellowships from FAPESP.

+Corresponding author: marsant2@yahoo.com

Received 17 August 2010

Accepted 8 December 2010 cyclic promastigotes then undergo apoptotic cell death by exposing phosphatidylserine (PS), thus participating in an altruistic inactivation of the macrophage host cells (Wanderley et al. 2009). Other promastigotes are phagocytised by the immune defence cells and initiate infection in the mammalian host.

The meta 1 gene was initially identified in Leishmania (Leishmania) major by differential complementary DNA library screening (Coulson \& Smith 1990) and was described to be expressed at a low steady-state level in procyclic promastigotes and amastigotes, compared to the high expression levels in metacyclic promastigotes (Nourbakhsh et al. 1996). Similar results were described for the meta 1 homologues in $L$. (L.) amazonensis and Leishmania (Leishmania) donovani (Uliana et al. 1999). Attempts at generating null mutants of this gene were unsuccessful, suggesting that it is an essential gene for parasite survival (Uliana et al. 1999). When over-expressed, the meta 1 protein induces an increase in the parasite virulence, both in the promastigotes and amastigotes (Uliana et al. 1999).

The use of reverse genetics allows for the generation of parasite mutants for studying the physiological roles of genes involved in infection (Beverley 2003). However, the characterization of the mutants, primarily with respect to their infectivity, sometimes fails because of the difficulties in properly comparing them to wild-type parasites. The purpose of this report was to study a genetic marker for which expression could be directly related to the infective-form percentage in a promastigote culture, which would allow us to compare the infectivity of different cultures or cultures at different stages by normalising the data to the number of infective cells.

We evaluated the in vitro infectivity of $L$. (L.) amazonensis along the culture growth curve related to the expression of meta 1 marker. We used peritoneal macrophages from $\mathrm{BALB} / \mathrm{c}$ mice, handled in accordance to the Institute of Biomedical Sciences, University of São Paulo, Animal Ethical Committee, for in vitro infections. To avoid variations in the experiments caused by 
infecting different macrophage preparations on different days of the growth curve using parasites from a single culture, we prepared a batch of promastigotes at different stages of culture to infect the same macrophage preparation at the same time.

Promastigotes at different stages of culture were obtained by starting a new culture every $24 \mathrm{~h}$ at a concentration of $5 \times 10^{5}$ cells $/ \mathrm{mL}$, using seed cells from the culture started the day before. The pattern of the curve obtained was similar to the one obtained using the conventional approach, in which the cell number of a single culture was determined every day (data not shown). Prior to starting the experiments, the cells were maintained at log phase by daily sub culturing for seven days to avoid variations in cell growth. Parasites recently recovered from mice were used in all experiments.

Total RNA, prepared by TRIzol $^{\circledR}$ extraction from cells at each culture stage, was used as the template in quantitative real-time reverse-transcription polymerase chain reaction (PCR). The mRNA copy-number of meta 1 , arginase and gapdh in each sample was determined by comparing the obtained cycle threshold with that obtained for a standard curve constructed using known amounts of the cloned PCR product of each target. The number of copies of the arginase and gapdh transcripts did not vary considerably along the growth curve. A melting curve was run for each PCR product to confirm the specificity of the reactions. The meta 1 mRNA level of each culture stage was normalised to the mRNA level of the housekeeping gene gapdh at the same stage by dividing the number of copies of meta 1 by gapdh (Fig. 1).

To ascertain the significance of the observed variations, the data were evaluated using analysis of variance. As expected, meta 1 was expressed at a low level in the early log phase, in agreement with the data from Uliana et al. (1999). Notably, the expression level of meta 1 in-

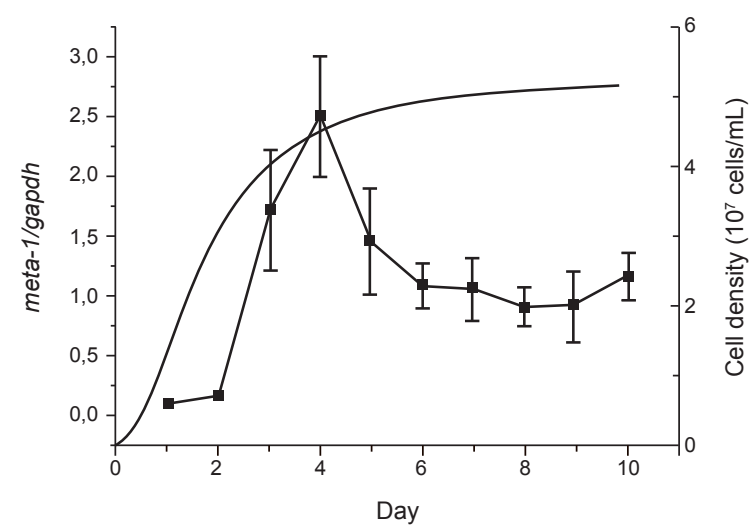

Fig. 1: meta 1 mRNA expression and Leishmania infectivity along the culture growth curve. Meta 1 and gapdh mRNA levels were determined by quantitative real-time reverse-transcription polymerase chain reaction from the total RNA obtained from cells at different days of culture. The dotted line represents the culture growth curve. The full line shows the meta 1 mRNA levels normalized to the gapdh mRNA levels for each day of culture and is a representative curve of three independent experiments. creased during the log phase, reaching the highest value at the end of this phase and at the beginning of the stationary phase (Fig. 1), when the culture was not expected to contain a large number of metacyclic cells (Sacks \& Perkins 1984). During the stationary phase, meta 1 expression decreased to a level between those found in the early and late log phases of the analyzed period.

Similar results were obtained when meta 1 expression was normalized to arginase expression (data not shown), which is another gene thought to be stably expressed across the different stages of differentiation (da Silva et al. 2002), in agreement with the pattern obtained from normalization to gapdh.

To investigate whether meta 1 expression correlates with the metacyclic percentage in the cultures, we determined the percentage of each cell subgroup by annexin- $\mathrm{V}$ and propidium iodide (PI) labelling, followed by flow cytometry (Fig. 2). The necrotic cells were detected by their PI fluorescence (Fig. 2A). The remaining cells were subdivided as follows: the annexin-V-positive cells were identified as the PS-exposing cells and the metacyclic cells were distinguished from the procyclic cells by their smaller size in the light forward scattering, as previously described (Wanderley et al. 2009). For the gate definitions, we took the cells in log phase with an almost exclusively procyclic population to define the cut-off point between metacyclic and procyclic cells (Fig. 2B). By defining the cut-off point on the boundary of the procyclic population, we are probably underestimating the total number of metacyclics in the culture, as there is a small overlap between the populations. However, this method ensured that we did not count procyclics as metacyclic cells and enabled us to quantify each subpopulation (Fig. 2C).

The proportion of procyclic, metacyclic, apoptotic and necrotic cell subgroups present at each stage of the culture growth curve can be seen in Table. The number of non-apoptotic metacyclic cells present in the cultures increased as the culture entered the stationary phase and remained constant at that stage. In addition, the number of necrotic and apoptotic cells increased during the late stationary phase (Table). The metacyclic percentage, determined at the late stationary phase when the cells were the most infective, was comparable to that in the insect host under natural conditions (Rogers et al. 2009).
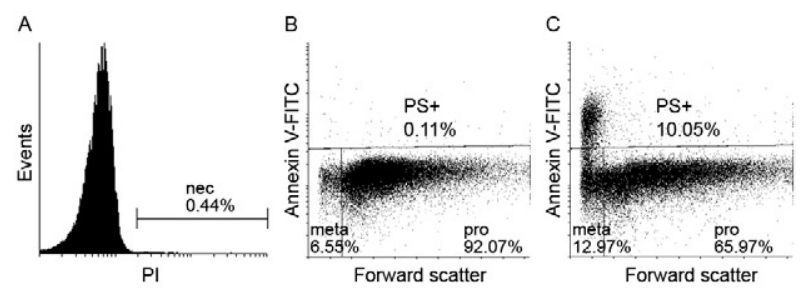

Fig. 2: flow cytometry-used gates and cell type proportion along the culture growth curve. A: the propidium iodide (PI) fluorescence histogram was used to detect the necrotic (nec) cells, which were excluded from the dot plots of forward scatter vs. annexin-V fluorescence (FITC); B: cells at day 3; C: cells at day 9; meta: metacyclic cells; pro: procyclic cells; PS+: annexin-V-labeled cells. 
TABLE

Infectivity index and cell type proportion

\begin{tabular}{lccccc}
\hline $\begin{array}{l}\text { Culture } \\
\text { day }\end{array}$ & $\begin{array}{c}\text { Infectivity } \\
(\mathrm{SD})\end{array}$ & $\begin{array}{c}\text { PS+ } \% \\
(\mathrm{SD})\end{array}$ & $\begin{array}{c}\text { Meta } \% \\
(\mathrm{SD})\end{array}$ & $\begin{array}{c}\text { Pro \% } \\
(\text { SD })\end{array}$ & $\begin{array}{c}\text { PI+ \% } \\
(\text { SD) }\end{array}$ \\
\hline 1 & $0.06(0.72)$ & $0.15(<0.01)$ & $6.56(0.05)$ & $91.17(0.04)$ & $0.71(<0.01)$ \\
2 & $0.08(0.97)$ & $0.15(<0.01)$ & $4.20(0.01)$ & $92.91(0.01)$ & $1.73(<0.01)$ \\
3 & $0.25(0.55)$ & $0.11(<0.01)$ & $6.55(0.01)$ & $92.07(0.01)$ & $0.44(<0.01)$ \\
4 & $0.37(0.61)$ & $0.17(<0.01)$ & $9.14(<0.01)$ & $89.37(0.01)$ & $0.45(<0.01)$ \\
5 & $0.68(0.46)$ & $0.25(<0.01)$ & $10.90(<0.01)^{a}$ & $87.48(<0.01)$ & $0.45(<0.01)$ \\
6 & $1.21(0.49)$ & $1.32(<0.01)$ & $10.17(0.01)^{a}$ & $79.85(<0.01)^{a}$ & $6.26(0.02)^{a}$ \\
7 & $2.97(0.29)^{a}$ & $3.66(0.01)^{a}$ & $10.36(0.01)^{a}$ & $76.25(0.05)^{a}$ & $8.69(0.03)^{a}$ \\
8 & $3.05(0.29)^{a}$ & $7.04(0.01)^{a}$ & $10.92(0.01)^{a}$ & $71.23(0.03)^{a}$ & $9.62(0.01)^{a}$ \\
9 & $2.37(0.36)^{a}$ & $10.05(0.03)^{a}$ & $12.97(0.02)^{a}$ & $65.97(0.05)^{a}$ & $9.80(0.04)^{a}$ \\
10 & $2.79(0.33)^{a}$ & $8.66(0.01)^{a}$ & $12.91(0.03)^{a}$ & $70.65(0.04)^{a}$ & $6.55(0.01)^{a}$ \\
\hline
\end{tabular}

$a: \mathrm{p}<0.05$, as compared to one, two and three-days-old cultures; infectivity: mean number of amastigotes per macrophage multiplied by the percentage of infected macrophages (a minimum of 200 macrophages from four independent experiments were analyzed for each point); meta: metacyclic cells; PI+: necrotic cells; Pro: procyclic cells; PS+: annexin-V-labeled cells; SD: standard deviation.

No direct correlation was observed between meta 1 expression and the metacyclic percentage in the cultures. The late log-phase peak of meta 1 expression overlapped with the time point at which the metacyclic percentage started to increase in the cultures. During the late stationary phase, the number of living metacyclic cells remained constant. Meta 1 expression also remained constant at a level lower than that observed in the late-log and early stationary phases. These data suggest that meta 1 expression is more important for the initiation of the differentiation process than for the maintenance of the metacyclic stage.

Next, we investigated whether meta 1 expression correlates with the ability of the parasites to infect macrophages in vitro. We infected murine peritoneal macrophages with $L$. (L.) amazonensis at different growth stages. The infectivity remained low during the log and early stationary phases and increased significantly during the stationary phase (Table).

Because the peak of meta 1 expression appeared at the late-log and early stationary phases, the obtained results revealed no direct correlation between meta 1 expression and cell infectivity, suggesting that meta 1 is expressed at a higher level when the cells are gaining infectivity, compared to when they are already infective. In contrast, infectivity correlated with the increase of apoptotic cells present in the culture (Pearson $\mathrm{r}=0.8433 ; 95 \%$ confidence interval 0.4555-0.9621; $\mathrm{p}<0.0025)$, supporting the importance of altruistic apoptotic cells for macrophage inactivation (van Zandbergen et al. 2006, Wanderley et al. 2006, 2009).

Previously, it has been shown that the stage-independent over-expression of the meta 1 gene enhances the infectivity of both promastigotes and amastigotes (Uliana et al. 1999). Notably, we detected no correlation between meta 1 expression and cell infectivity or the number of metacyclic cells present in culture. However, meta 1 did overlap with the phase in which the metacyclic percentage in culture started to increase. This result suggests that meta
1 might be involved in cell differentiation from the procyclic form into the metacyclic form, preparing the cells for the infective form in the insect host, and that it could be an indicator of the differentiation rate in the culture.

In this study, we showed that the expression profile of the meta 1 gene was more complex than expected from the existing data, which evaluated only the mid-log and stationary phases. This finding would not be obtained by analysing only two points of the growth curve and many interesting results would be missed using that approach. The gene expression analysis at each day of the culture growth curve revealed an orchestrated pattern of the meta 1 expression peak followed by a surge in the metacyclic percentage in culture, leading to an increase in cell infectivity. This observation suggests that meta 1 may be an attractive target for further studies regarding its role in metacyclogenesis. Meta 1 is highly conserved among the subgenus Leishmania (Leishmania) and Leishmania (Viannia) (Berberich et al. 1998) and this degree of conservation could indicate a critical role in the parasite life cycle.

Further studies are required to identify an easily detected genetic marker for indicating culture infectivity. However, the determination of apoptotic metacyclic percentage by flow cytometry analysis can be used in comparative studies of mutant phenotypes regarding their infectivity in mammalian cells.

\section{REFERENCES}

Bates PA, Tetley L 1993. Leishmania mexicana: induction of metacyclogenesis by cultivation of promastigotes at acidic pH. Exp Parasitol 76: 412-423.

Berberich C, Marín M, Ramírez JR, Muskus C, Vélez ID 1998. The metacyclic stage-expressed meta 1 gene is conserved between Old and New World Leishmania species. Mem Inst Oswaldo Cruz 93: 819-821.

Beverley SM 2003. Protozomics: trypanosomatid parasite genetics comes of age. Nat Rev Genet 4: 11-19. 
Coulson RM, Smith DF 1990. Isolation of genes showing increased or unique expression in the infective promastigotes of Leishmania major. Mol Biochem Parasitol 40: 63-75.

Cunningham AC 2002. Parasitic adaptive mechanisms in infection by Leishmania. Exp Mol Pathol 72: 132-141.

da Silva ER, Castilho TM, Pioker FC, Tomich de Paula Silva CH, Floeter-Winter LM 2002. Genomic organisation and transcription characterisation of the gene encoding Leishmania (Leishmania) amazonensis arginase and its protein structure prediction. Int J Parasitol 32: 727-737.

Descoteaux A, Turco SJ 1999. Glycoconjugates in Leishmania infectivity. Biochim Biophys Acta 1455: 341-352.

Franke ED, McGreevy PB, Katz SP, Sacks DL 1985. Growth cycledependent generation of complement-resistant Leishmania promastigotes. J Immunol 134: 2713-2718

Nourbakhsh F, Uliana SR, Smith DF 1996. Characterisation and expression of a stage-regulated gene of Leishmania major. Mol Biochem Parasitol 76: 201-213.

Rogers M, Kropf P, Choi BS, Dillon R, Podinovskaia M, Bates P, Müller I 2009. Proteophosophoglycans regurgitated by Leishmaniainfected sand flies target the L-arginine metabolism of host macrophages to promote parasite survival. PLoS Pathog 5: e1000555.
Sacks DL, Hieny S, Sher A 1985. Identification of cell surface carbohydrate and antigenic changes between noninfective and infective developmental stages of Leishmania major promastigotes. J Immunol 135: 564-569.

Sacks DL, Perkins PV 1984. Identification of an infective stage of Leishmania promastigotes. Science 223: 1417-1419.

Uliana SR, Goyal N, Freymüller E, Smith DF 1999. Leishmania: overexpression and comparative structural analysis of the stageregulated meta 1 gene. Exp Parasitol 92: 183-191.

van Zandbergen G, Bollinger A, Wenzel A, Kamhawi S, Voll R, Klinger M, Müller A, Hölscher C, Herrmann M, Sacks D, Solbach W, Laskay T 2006. Leishmania disease development depends on the presence of apoptotic promastigotes in the virulent inoculum. Proc Natl Acad Sci USA 103: 13837-13842.

Wanderley JL, Moreira ME, Benjamin A, Bonomo AC, Barcinski MA 2006. Mimicry of apoptotic cells by exposing phosphatidylserine participates in the establishment of amastigotes of Leishmania $(L)$ amazonensis in mammalian hosts. J Immunol 176: 1834-1839.

Wanderley JL, Pinto da Silva LH, Deolindo P, Soong L, Borges VM, Prates DB, de Souza AP, Barral A, Balanco JM, do Nascimento MT, Saraiva EM, Barcinski MA 2009. Cooperation between apoptotic and viable metacyclics enhances the pathogenesis of Leishmaniasis. PLoS One 4: e5733. 